\title{
Quantum-Relativistic Effects at the Nanolevel: Analysis of the Mean Square Deviation of Position with a new Analytical Model
}

\author{
Paolo Di Sia ${ }^{1-3}$ \\ ${ }^{1}$ University of Padova, School of Science \& School of Medicine, Department of \\ Neuroscience, Via Jappelli 1, I-35121 Padova, Italy \\ ${ }^{2}$ Email: paolo.disia@gmail.com \\ ${ }^{3}$ Webpage: www.paolodisia.com
}

\begin{abstract}
In this paper I present new results about the quantum-relativistic form of the mean square deviation of position related to a recently appeared Drude-Lorentz-like model already performed in classical and quantum way, named DS model. The model gives precise informations about the distance traveled by a carrier (electrons, ions, etc.) inside a nanostructure in the hypothesis of quantum effects and relativistic velocity. It has a wide scale range of applicability; I consider the nanoscale in this paper, but the model holds from sub-pico-level to macro-level because of the presence of a gauge factor, making it applicable to all oscillating processes in Nature. Examples of application and suggestions complete the paper.
\end{abstract}

Keywords: DS model; Drude-Lorentz-like Models; Mean Square Deviation of Position; Nanoscience; Quantum-Relativistic Effects; Surface Dynamics; Solid-State Physics; Analytical modelling.

\section{References:}

[1] P. Di Sia, An Analytical Transport Model for Nanomaterials, Journal of Computational and Theoretical Nanoscience, 8, 84-89, 2011.

[2] P. Di Sia, An Analytical Transport Model for Nanomaterials: The Quantum Version, Journal of Computational and Theoretical Nanoscience, 9, 31-34, 2012. 
[3] P. Di Sia, Oscillating velocity and enhanced diffusivity of nanosystems from a new quantum transport model, Journal of Nano Research, 16, 49-54, 2011.

[4] P. Di Sia, A new theoretical method for transport processes in nanosensoristics, Journal of Nano Research, 20, 143-149, 2012.

[5] P. Di Sia, Nanotechnology between Classical and Quantum Scale: Applications of a new interesting analytical Model, Advanced Science Letters, 5, 1-5, 2012.

[6] P. Di Sia, About the Influence of Temperature in Single-Walled Carbon Nanotubes: Details from a new Drude-Lorentz-like Model, Applied Surface Science, 275, 384-388, 2013.

[7] P. Di Sia, Characteristics in Diffusion for High-Efficiency Photovoltaics Nanomaterials: an interesting Analysis, Journal of Green Science and Technology, 1, 1-4, 2014.

[8] P. Di Sia, Nanobiomaterials for environmental protection: state of the art, applications and modelling, International Journal of Engineering Innovations and Research (IJEIR), 3(5), 688-693, 2014.

[9] P. Di Sia, An New Analytical Model for the Analysis of Economic Processes, Theoretical Economics Letters, 3(4), 245-250, 2013.

[10] F. Dyson, Adavanced Quantum Mechanics, Singapore: World Scientific Publishing, 2007.

[11] W. Rudin, Real and Complex Analysis, New York: McGraw-Hill Higher Education, $3^{\text {rd }}$ ed., 1987.

[12] P. J. Ventura, L. C. Costa, M. C. Carmo, H. E. Roman, L. Pavesi, AC Conductivity of Porous Silicon from Monte Carlo Simulations, Journal of Porous Materials, 7(1-3), 107-110, 2000.

[13] J. B. Baxter and C. A. Schmuttenmaer, Conductivity of $\mathrm{ZnO}$ Nanowires, Nanoparticles, and Thin Films Using Time-Resolved Terahertz Spectroscopy, Journal of Physical Chemistry B, 110, 25229-25239, 2006.

[14] J. B. Baxter, and C. A. Schmuttenmaer, Carrier Dynamics in Bulk ZnO. I. Intrinsic Conductivity Measured by Terahertz Time Domain Spectroscopy, Physical Review B, 80, pp. 235206-1, 2009. 
[15] C. A. Schmuttenmaer, Using Terahertz Spectroscopy to Study Nanomaterials, Terahertz Science and Technology, 1(1), 1-8, 2008.

[16] S. L. Dexheimer (Ed.), Terahertz Spectroscopy: Principles and Applications, Boca Raton: CRC Press Taylor \& Francis Group LLC, 2008.

[17] M. C. Beard, G. M. Turner, and C. A. Schmuttenmaer, Terahertz spectroscopy, Journal of Physical Chemistry B, 106(29), 7146-7159, 2002.

[18] P. Di Sia, Relativistic nano-transport and artificial neural networks: details by a new analytical model, International Journal of Artificial Intelligence and Mechatronics (IJAIM), 3(3), 96-100, 2014.

[19] B. Barwick, D. J. Flannigan \& A. H. Zewail, Photon-induced near-field electron microscopy, Nature, 462, 902-906, 2009, doi: 10.1038 / nature08662.

[20] F. Borondics, K. Kamarás, M. Nikolou, D. B. Tanner, Z. H. Chen, and A. G. Rinzler, Charge dynamics in transparent single-walled carbon nanotube films from optical transmission measurements, Physical Review B, 74, 045431-045436, 2006.

[21] K. El Shabrawy, K. Maharatna, D. Bagnall, B. M. Al-Hashimi, Modeling SWCNT Bandgap and Effective Mass Variation Using a Monte Carlo Approach, IEEE Transactions on Nanotechnology, 9(2), 184-193, 2010.

[22] M. Th Hassan, Attomicroscopy: from femtosecond to attosecond electron microscopy, Journal of Physics B: Atomic, Molecular and Optical Physics, 51, 032005, 2018.

[23] K. Bennett, J. R. Rouxel, and S. Mukamel, Linear and nonlinear frequency- and time-domain spectroscopy with multiple frequency combs, Journal of Chemical Physics, 147, 094304, 2017, doi: http://dx.doi.org/10.1063/1.5000375.

[24] H. A. Hafez, X. Chai, Y. Sekine, M. Takamura, K. Oguri, I. Al-Naib, M. M. Dignam, H. Hibino, and T. Ozaki, Effects of environmental conditions on the ultrafast carrier dynamics in graphene revealed by terahertz spectroscopy, Physical Review B, 95, 165428, 2017.

[25] P. Di Sia, A new analytical transport model for (nano)physics, International Research Journal of Engineering and Technology (IRJET), 2(7), 1-4, 2015.

[26] P Di Sia, Quantum-Relativistic Carrier Nano-Transport and Plasmonics, Functional Nanostructures Proceedings, 1(1), 67-69, 2017. 
[27] P. Di Sia, Quantum-Relativistic Velocities in Nano-Transport, Applied Surface Science, 446, 187-190, 2018, https://doi.org/10.1016/j.apsusc.2018.01.273.

[28] P. Di Sia, Mathematics and Physics for Nanotechnology - Technical Tools and Modelling, Singapore: CRC Press Taylor \& Francis Group LLC, 2019.

https://www.crcpress.com/Mathematics-and-Physics-for-Nanotechnology-Technical-Tools-and-Modelling/Sia/p/book/9789814800020 